\title{
On the Mismeasurement of Impulsivity: Trait, Behavioral, and Neural Models in Alcohol Research among Adolescents and Young Adults
}

\author{
Kevin M. King • Julie A. Patock-Peckham • \\ Alecia D. Dager • Kristine Thimm • Jonathan R. Gates
}

Published online: 4 January 2014

(C) Springer International Publishing AG 2014

\begin{abstract}
We review the extent to which the psychosocial trait, behavioral, and neural models of impulsivity in adolescence and young adulthood commonly used in alcohol research may be reconciled. Recent advances in the development of trait models of impulsivity have converged towards a three-trait framework of impulsigenic traits, with motivational drive (extraversion), effortful control (conscientiousness and/ or constraint), and emotion dysregulation (neuroticism) at its core, which has similar developmental models in childhood. Behavioral models of impulsivity have focused on a twofactor model centered around impulsive choice versus impulse response, which seems to have little theoretical or empirical connection with trait models of impulsivity. Throughout, we review recent neuroimaging research related to trait and behavioral impulsivity. We also discuss research on how trait
\end{abstract}

\author{
K. M. King $(\bowtie) \cdot K$. Thimm \\ Department of Psychology, University of Washington, Box 351525, \\ Seattle, WA 98195, USA \\ e-mail:kingkm@uw.edu \\ K. Thimm \\ e-mail: kthimm@uw.edu
}

J. A. Patock-Peckham · J. R. Gates

Department of Psychology, Arizona State University, Box 871104, Tempe, AZ 85287, USA

J. A. Patock-Peckham

e-mail: julie.patock@asu.edu

J. R. Gates

e-mail: jrgates1@asu.edu

A. D. Dager

Department of Psychiatry, Yale University,

300 George St., Suite 901, New Haven, CT 06511, USA

e-mail: alecia.dager@yale.edu

A. D. Dager

Olin Neuropsychiatry Research Center, Institute of Living at

Hartford Hospital, 200 Retreat Ave, Hartford, CT 06106, USA and behavioral impulsivity are associated with alcohol use risk and AUDs and how behavioral impulsivity is affected by alcohol use. We note significant problems in integrating trait and behavioral research, driven by the absence of a shared theoretical framework for behavioral and trait models, a general underrepresentation of developmental research, and inconsistencies in definitions of impulsivity in the literature.

Keywords Impulsivity · Disinhibition · Self-control · State-trait models $\cdot$ fMRI $\cdot$ Neural imaging $\cdot$ Alcohol-use disorders $\cdot$ Alcohol involvement

\section{Introduction}

Alcohol-use disorder is characterized by impaired control over alcohol use (i.e. the inability to stop drinking despite intentions to do so [1]), which often leads to harmful outcomes [2, $3 \cdot \bullet$. Impaired control may capture an impulsivity to compulsivity shift in addiction to alcohol that reflects a cross-over in motivation from positive to negative reinforcement [4]. Children and adolescents who are observed to have difficulty controlling problematic behavior before drinking begins are known to be at risk of alcohol-use disorders $[5,6]$, and problems with behavioral control have been crosssectionally linked to difficulty controlling alcohol use among young adults $[7,8]$, suggesting the same brain mechanisms that go awry in alcohol disorders may also predispose adolescents and young adults to those disorders in the first place. Indeed, the neurobiological basis of this impaired control has become better understood in recent years, with research focusing on the neural substrates of addiction in terms of dysfunction in regions of the brain related to constraint and reward [9].

Alcohol use increases dramatically from adolescence to young adulthood, with $11 \%$ of 8th graders in the United 
States being past-month drinkers to $42 \%$ of 18 year olds being past-month drinkers [10] and by age $20,45.5 \%$ of individuals report binge drinking (consuming five or more drinks) at least twice a month [11]. During this same period, regions of the adolescent brain responsible for self-control are being dramatically reorganized; this involves increases in white matter volume and integrity, reflecting myelination and increased fiber organization, and reductions in gray matter volume associated with synaptic pruning [12-14]. Moreover, there is rapid maturation of mesolimbic dopamine systems, including the nucleus accumbens and the amygdala, which occurs around puberty, resulting in high novelty-seeking, reward sensitivity, and risk taking during adolescence which declines into young adulthood [15]. In contrast, the prefrontal regions which underlie inhibitory control and decision making develop relatively late $[13,16]$. This pattern of brain development produces a developmental "asymmetry", with high motivation for reward developing early in adolescence and general impulse control developing late, increasing the propensity to engage in risky behavior during adolescence relative to adulthood $[17,18]$. These developmental changes in the brain have been shown to be accompanied by changes in trait measures of sensation seeking (for the dopamine systems) and impulse control and self-control (for inhibitory control systems) [19•, 20]. Thus, it is critical to understand how existing models of impulsivity may be mapped on to these brain regions that are so important to adolescent development.

Similar to definitions of addiction, impulsive behavior is one without forethought or regard for subsequent consequences, and "impulsigenic traits" can be thought of as traits that produce a tendency to regularly engage in impulsive behavior $[21,22 \cdot, 23]$. Yet impulsivity is so often inconsistently defined in studies it is difficult to draw firm conclusions about what we do and do not know regarding the development and maintenance of addiction, except that less impulsivity is good and more is bad. This review attempts to combine models of impulsivity commonly utilized in alcohol research among adolescents and emerging adults from a psychosocial trait, behavioral, and neurobiological perspective, to provide an integrated perspective on matters of impulsivity and alcohol.

\section{Trait Models of Impulsivity}

Impulsivity is often measured as a trait by asking people to rate themselves on specific behavior or characteristics (such as "I often act on impulse" or "I often act without thinking" or "I often do things on the spur of the moment", [23]). In one sense, these trait measures are assessing individual's perceptions of the causes of their impulsive behavior, in that they are asking a person to look back over aggregations of their behavior and rate the degree to which they acted "on the spur of the moment" vs. "without thinking" vs. "acted on impulse". More recent researchers then labeled aggregations of these items impulse control, planning, sensation seeking, impulsivity, urgency, good or bad impulsivity, or with some related label [23-27]. These measures can tell us who is at risk of alcohol use, problems, and disorders, and indeed, children, adolescents and young adults who rate themselves as more impulsive or who are rated by others as more impulsive have been consistently shown to be associated with alcohol use and problems (recently reviewed elsewhere [28•, 29•, $30 \bullet]$ ). Trait measurement is fast, generally reliable, and can thus be conducted for large numbers of respondents over long periods of time.

Indeed, just as high sensation seeking and low impulse control in adolescence are two different explanations of the causes of impulsivity, many traits have been proposed to explain impulsive behavior. In the past decade the field has been converging on a model of impulsivity with three components $[22 \bullet, 31 \bullet \cdot, 32 \bullet \cdot]$. This model is similar to the UPPS trait model of Whiteside and Lynam [27] and Smith et al. [26] and also fits Carver's [21] biologically based model which bridges Gray's reinforcement sensitivity theory with developmental theory [33]. Table 1 illustrates this model with sample item domains from representative scales that cover each domain (note these scales are not meant to represent all scales that could be used to represent each domain).

This first trait has been described as extraversion/positive emotionality $(\mathrm{E} / \mathrm{PE})[32 \cdot \bullet]$ which is also associated with multiple measures of sensation and novelty seeking, and reward sensitivity [21,34]. This trait has strong similarities with the behavioral activating system $[24,35]$, which is an activating system producing impulses towards behavior, thought to reflect activity in reward centers in the brain (as noted above, sensation seeking accompanies reward system development throughout adolescence). Moreover, dopamine receptor availability in the striatum has been associated with individual differences in sensation seeking [36, 37], and broad extraversion has been correlated with volume of brain regions associated with reward processing [38•]. Sensation seeking has consistently been shown to be weakly correlated with other facets of impulsivity $[22 \bullet, 31 \bullet \cdot$, and in a recent review a "meta-factor" of E/PE was uncorrelated to moderately correlated $(r=0.22)$ with another facet of impulsivity [32••].

The second trait indicates broad conscientiousness, planning, thinking before acting, and perseverance. It is commonly measured among children (i.e. "effortful control" [33, 39, 40]), adolescents (impulse control, planning, perseverance $\left[19 \bullet, 30^{\bullet}\right]$ ), and young adults (planning, perseverance, broad conscientiousness [26]) with different labels that describe very similar ideas. This trait is also reflected in Carver's 2005 model of impulsivity, and extensively throughout the child temperament literature [33], and reflects attention focusing and shifting and inhibition, and is also labeled disinhibition 
Table 1 A three factor model of impulsivity, with sample items for typical measures and relationships with alcohol outcomes

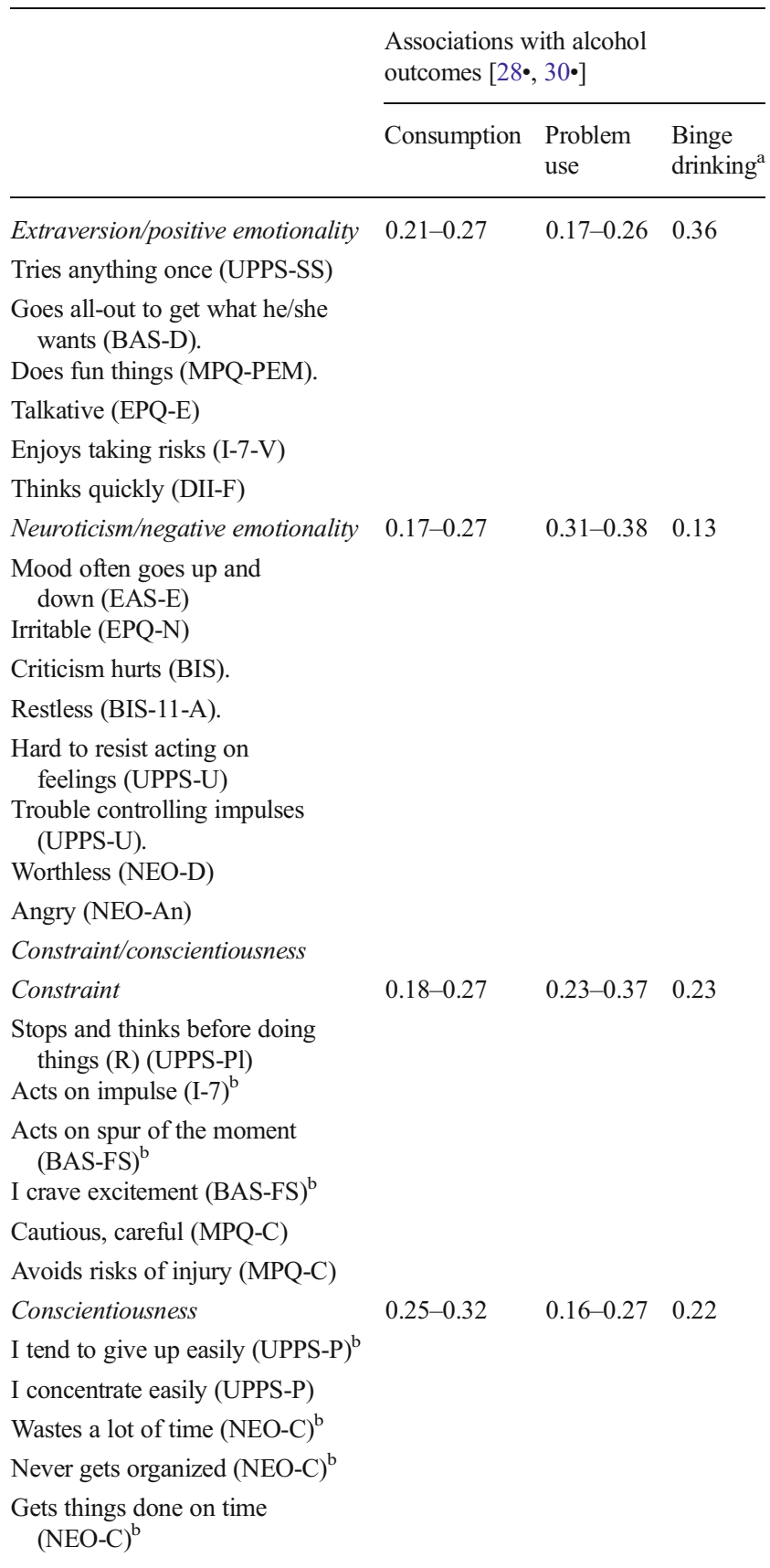

UPPS impulsive behavior scale [27]; BIS/BAS, = behavioral inhibition/ behavioral activation system [149]; MPQ, = multidimensional personality questionnaire [150]; Eysenck personality questionnaire [151]; Eysenck impulsiveness questionnaire [23]; Dickmann impulsiveness inventory [152]; EAS, = emotionality, activity level, and sociability [153]; NEO, = NEO personality inventory, revised [154]

${ }^{\text {a }}$ Binge drinking findings taken from Ref. [28•] only

${ }^{\mathrm{b}} \mathrm{R}$ indicates domain loads in reverse of construct label

Extraversion: SS, sensation seeking; D, drive; PEM, positive emotionality; E, extraversion; V, venturesomeness; F, functional impulsivity

Neuroticism: E, emotionality; N, neuroticism; A, attentional impulsivity;

$\mathrm{U}$, negative urgency; $\mathrm{D}$, depression; An, anger

Constraint: Pl, planning; FS, fun-seeking; $\mathrm{C}$, constraint

Conscientiousness: $\mathrm{P}$, persistence; $\mathrm{C}$, conscientiousness versus constraint conscientiousness (DvC/C) impulse control, planning and/or premeditation, perseverance and/or persistence, conscientiousness, good self-control, and future orientation in the adolescent and young adult alcohol literature [21, 23, 26, 27, 32••, 41-43]. However, not all of these scales share identical item content within each scale. For example, one popular measurement used to validate theories of adolescent brain development, the impulse control subscale of the Weinberger adjustment inventory (WAI; [44]), confounds sensation seeking and effortful control. Several authors have noted this "jingle" fallacy in reviews of the literature [27, $32 \bullet \bullet$, where scales with similar names have different content at the item level. In the UPPS model, Whiteside and Lynam [27] disaggregated this factor into two facets, (lack of) planning and (lack of) perseverance. A recent meta-analysis confirmed this distinction, suggesting that scales reflecting acting without thinking (such as UPPS planning) and acting on impulse (for example the Eysenck $I 7$ impulsivity) load on one distinct facet that can be regarded as "effect free", called disinhibition vs. constraint, whereas scales reflecting (lack of) persistence and poor self-discipline loaded on a moderately $(r=0.55)$ correlated factor that also had loadings with EPI neuroticism and emotionality, labeled conscientiousness and/ or will vs. resourcelessness [32••]. Although many researchers have speculated that this component of impulsivity is largely driven by activity in the pre-frontal cortex, very little research has connected individual differences in trait measures with individual differences in neural structure or function. In one study, higher parent and teacher ratings on the parent behavior scale (including items combining content from effortful control and impulsivity scales, for example "impulsive, acts without stopping to think" and such symptoms of ADHD as "hyperactive, always on the go") were associated with lower volumes of the right ventromedial prefrontal cortex among 67 children and adolescents from the community [45]. In another study, larger prefrontal cortex volumes were associated with greater conscientiousness [38•].

The third trait is another effect-laden facet which reflects rash decision making paired with negative emotionality. Sharma et al. [32••] labeled this factor neuroticism and/or negative emotionality which is measured by use of such terms as negative urgency, neuroticism, and negative emotionality. Other scales which accompany this include poor self-control, emotional control, aggression control, and present-hedonistic $[21,23,25,26,43,46]$. This aspect is also frequently observed in the child literature but is not described as a component of impulsivity, but rather a distinct aspect of temperament described as "negative emotionality" [33, 39]. This trait may reflect the functioning of what Gray described as the behavioral inhibition system (reviewed elsewhere [35, 47]) a "conflict detection and resolution system" that attempts to resolve conflicting signals about reward or punishment. The updated reinforcement sensitivity theory links the functioning 
of the BIS closely with neuroticism and negative emotionality $[35,47]$.

The neural basis of this trait has only recently been investigated. Boy et al. [48] suggested GABA receptor concentration in the prefrontal cortex was associated with negative urgency. One recent large $(n=265)$ multi-modal brainimaging study found neuroticism was associated with smaller total brain volume and a smaller fronto-temporal surface area, which may reflect the effect of early life stress on development or the degree of stress sensitivity in individuals with high neuroticism [49]. Another study found an association between neuroticism and reduced volumes in regions of the brain implicated in threat detection, goal conflict, error detection, and sensitivity to punishment, for example in the dorsomedial $\mathrm{PFC}$, the posterior hippocampus, and portions of the basal ganglia and midbrain [38•].

Not all researchers have accepted or utilized this framework. Many still utilize a single "impulsiveness" factor reflecting a broad inability to modify reward drive-initiated behavior despite negative consequences, measured by broadband scales such as the Eysenck I7 impulsivity scale, the BIS11 total score, novelty seeking, and MPQ-constraint [34, 50]. This approach to characterizing impulsivity is somewhat similar to the "developmental asymmetry" model of adolescence described above $[17,18,51 \bullet]$, which utilizes consistent measures of sensation seeking, but often utilizes measures of impulse control (i.e. [44]) that mix sensation seeking and constraint [52]. Generally, there has been little developmental research on how this third facet of impulsivity may change throughout adolescence in relation to extraversion and/or positive emotionality or effortful control.

Reviews of the literature are indicative of mixed findings on the relationship between broad extraversion and neuroticism and alcohol use and AUDs [53]. However, sensation seeking has been consistently associated with alcohol use and problems, with recent meta-analyses suggesting that it has its strongest effects on involvement in alcohol use and binge drinking, while negative urgency has strong associations with alcohol-related problems and AUDs [28•, 30•]. Some authors have suggested that sensation seeking (reflecting sensitivity to positive reinforcement) is associated with the early stages of alcohol involvement, but not necessarily the progression to abuse or dependence [54••]. Similarly, it may be that negative urgency (i.e. sensitivity to negative reinforcement) is involved in the development of dependence as alcohol use escalates, impaired control over alcohol use emerges, and alcohol use becomes compulsive [54••]. Thus it may be that mixed findings of previous studies of extraversion or neuroticism have either been because of a focus on the wrong stage of alcohol use or AUD or because more specified measures of extraversion that reflect sensitivity to reward (for example sensation seeking) or neuroticism (that measure negative urgency specifically) are the best predictors of alcohol involvement rather than general extraversion or neuroticism. On the other hand, general measures of conscientiousness and constraint have been consistently associated with alcohol use in the literature [53]. Meta analyses suggest that a lack conscientiousness is associated with both involvement in and problems with alcohol use in adolescence [30॰ and young adulthood and beyond [28 ], but a lack of conscientiousness (in these meta-analyses assessed with the UPPS-perseverance scale) was most strongly associated with alcohol use, and somewhat less so with problem use or binge drinking. Conversely, low constraint seems to be a general risk factor for alcohol involvement, predicting both use and problems at moderate levels across studies $[28 \bullet, 30 \bullet$.

\section{Behavioral Models of Impulsivity}

Behavioral models provide an ideal framework for testing hypotheses about when and how individuals are at risk of alcohol problems. This is impulsivity measured as a behavior, such as whether or not an individual can suppress a keystroke response or an eye saccade when given a "stop" signal in a go/ no go situation, suppress an automatic response to read a word to describe the color of a word (as in a Stroop task), or how likely someone is to choose between rewards that appear sooner but are smaller than those which appear later $[20$, 55-57]. Although these behavioral measurements are often intended to capture something about impulsigenic traits, because impulsive actions or choices are directly observed, situational effects may also be measured (such as in an experimental setting), and thus these measures can also capture state variability in impulsivity. Moreover, behavioral measures can also be deployed in neurobiological settings (for example use of an fMRI or PET scanner) to investigate the neural factors underlying performance on these tasks.

Similar to trait impulsivity, researchers have examined multiple causes of impulsivity at the behavioral level. These tasks can be broadly classified into two categories [22, $31 \bullet$, $58,59]$, which has been described as a dichotomy of impulsive choice versus impulsive responding (sometimes also called impulsive action) [60]. Impulsive response tasks are affected by executive cognitive function (ECF, i.e. working memory, planning, attention, and response inhibition [61]), and use the ability to the ability to rapidly initiate or inhibit a response (i.e. go/no go, stop signal, continuous performance tasks (CPT), Stroop tasks). Although alcohol researchers more narrowly focus on response inhibition or initiation, there is also a wider range of what Cross et al. [31 ••] called "visual cognitive" tasks that seem to require broader executive function, for example the tower of London, trails, and Porteus maze tests, which require the integration of the multiple domains of ECF. 
On the other hand, impulsive choice tasks assess decision making and risky choice, In impulsive choice models, preference for reward is commonly assessed in two ways. Delay discounting models manipulate the probability, size and time to reward, assessing individuals' preference for delayed versus immediate rewards in a range from certainty to uncertainty (i.e. delay discounting task and two-choice task, [55, 62]). More complex reward models balance this explicit or implicit information about the size and probability of a potential reward against the size and probability of potential losses (i.e. Iowa gambling task (IGT) [63], Columbia card task (CCT) [64], or the balloon analogue risk task (BART) [65]). All of these tasks provide trial by trial feedback about gains and losses, allowing participants to adjust their behavior to optimize gains and minimize losses, but they vary in the extent to which this information is provided explicitly (for example in the CCT) or implicitly (for example in the BART). Some authors have also described these tasks as "reflection impulsivity", describing an individuals' ability to integrate information about complex decisions [66], which acknowledges that these tasks seem to capture both sensitivity to gains and losses and overall risk-taking strategies that affect behavioral choices. Examples of these tasks are provided in Table 2.

However, theoretical categorizations of behavioral tasks are not consistent throughout the literature. For example, some authors have argued that go/no-go, CPT, antisaccade tasks, and go-stop tasks all measure response inhibition $[67,68 \bullet, 69$, 70]. Others have suggested that the go/no-go task and CPT, in which trials alternate between "go" and "stop" cues measure something different and distinct from response inhibition tasks, in which a single trial may include a "go" cue followed by a "stop" cue [71, 72]. The Stroop task has been categorized both as a measure of response inhibition, in which participants must inhibit an automatic response in favor of the appropriate response, and a task measuring resistance to distracting information, similar to a Flanker task, which presents conflicting information with a target stimulus [56]. Furthermore, empirical data have not always supported theoretical categorizations. Tasks purported in some frameworks to measure response inhibition have been shown to be uncorrelated in factor analysis [32••, 55]. And, of course, the converse has also been found [73], suggesting that some tasks either assess multiple facets of impulsivity or that performance in more complex tasks is subserved by the abilities measured in other tasks.

Moreover, within the same behavioral task, for example a Stroop task or a stop-signal task, researchers will often use a different outcome from the task in different studies. For example, in executive function tasks requiring rapid reaction time and rapid decision making (for example CPT, go/no go, Stroop, stop-signal tasks), performance in some tasks can be measured by either reaction times or errors made. Even within these categories, both reaction time and errors can be calculated in different ways. For example, to indicate inhibition, some research has used the number of errors whereas others have used a ratio of errors to correct responses to represent impulsive responding [74, 75]. Reaction times can also be measured in terms of average latencies on either "go" or "stop" trials and the difference between the delay time at which participants can inhibit their responses $50 \%$ of the time on "stop" trials and the mean reaction time on "go trials" [59]. However, it may be that some outcomes measure impulsive behavior more accurately than others, and some outcomes may assess different neurocognitive processes entirely. Specifically, researchers have suggested omission errors (i.e. not responding when prompted) may indicate sustained attention, response time may reflect information-processing requirements, and commission errors (i.e. inappropriate responding) alone assess impulsive responding [76]. Despite these distinctions, all of these outcomes have been assumed to be indicative of the same concept, and little work has been conducted to determine whether different aspects of the same task capture similar or different self-control mechanisms.

Presumably, results from impulsive response tasks are indicative of inhibition [77], and are undergirded by the function of the anterior cingulate cortex (ACC) function and other prefrontal cortex (PFC) components [78]. Several response inhibition tasks, for example the go/no-go and stop signal reaction time tasks, have been used in rodent studies, and findings in rodent and human studies have been remarkably consistent [79]. These studies not only reveal the function of the PFC in response inhibition, but also identify the right inferior frontal gyrus as a region of interest [80]. Moreover, there is mounting evidence that there may be a front-striatal circuit involved in stopping behavior, with research suggesting that this circuit explains poor performance in inhibition tasks among children with ADHD [81].

It should also be noted that important critiques have called these assumptions into question [82, 83••, 84•]. First, inhibition tasks tend to load on broad executive function factors rather than a specific inhibition factor $[83 \bullet \bullet, 85]$. Others have shown that effortful control involves the executive function of updating and monitoring information in working memory but does not involve inhibition [86]. Moreover, investigators [82, 84.] suggest the function of the PFC is to manage multiple objective-relevant processing pathways, and inhibition "failures" arise because of an inability to either detect a signal that a second objective-relevant pathway has arisen or of an inability to manage a weaker objective-relevant signal. This framework of inhibition has been described not as a "don't do X", but rather a "do Y" framework, similar to response inhibition concerning choices between two or more alternative "do Y" pathways relevant to appropriate contextual cues [84•].

Risky decision making on the IGT is believed to be subserved, in part, by the ventro-medial prefrontal cortex (vmPFC/ACC) [87]. This region is believed to be involved 


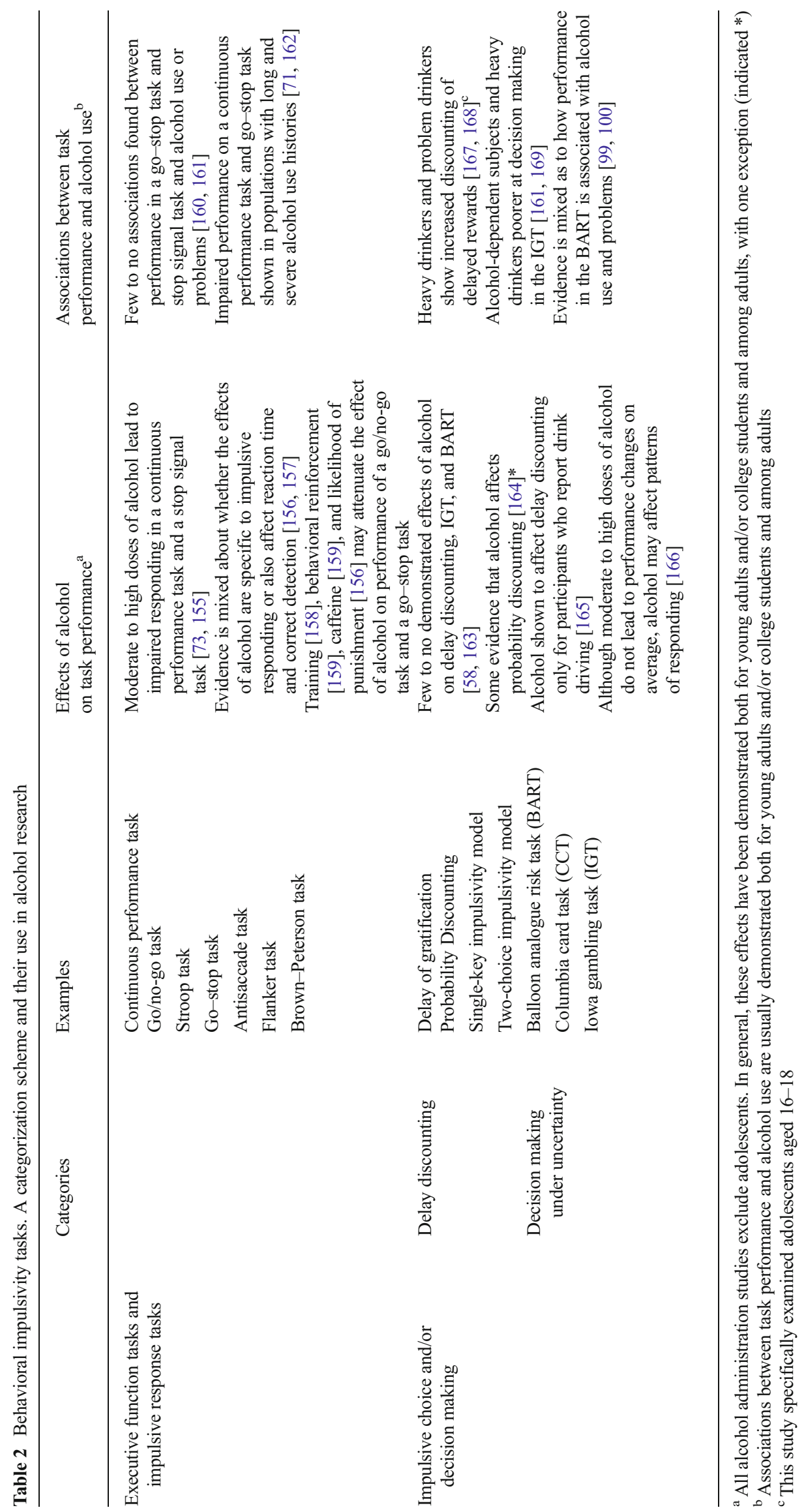


in assessing uncertainty, with activation typically increased as risk increases and prefrontal control regions being engaged as risky choices are inhibited [88-91]. Among adults with AUD, higher alcohol problem scores were associated with lower dorsal $\mathrm{mPFC}$ response, which could indicate a deficiency in error prediction and risk avoidance, contributing to continued risky drinking [92•]. Among emerging adults, diminished response of this region during risky choices was associated with alcohol use and with higher scores on the Zuckerman sensation seeking scale, suggesting that heavier drinking and disinhibited individuals fail to disengage from risky decisions [93]. This work provides evidence of overlap between trait disinhibition, reward-seeking brain response, and alcohol use.

Behavioral tasks have been commonly used in imaging studies, alcohol administration studies, and alcoholassociation studies in the alcohol literature (Table 2), with imaging studies often being combined with the last two. The findings outlined in Table 2 show that alcohol intoxication affects impulsive responding more than impulsive choices. Imaging studies seem to provide conflicting findings about how alcohol use affects inhibition. One study of heavydrinking adolescents revealed greater activation during a gono/go task in regions associated with response inhibition (for example the left and right middle frontal gyri and the right inferior parietal lobe) [94•]. Another study among college students found the opposite: in a go-no/go task, less activation in the left and right middle frontal gyri and the left and right parietal lobes (and other locations) was observed for heavy drinkers, but only when they correctly inhibited a response [95]. On the other hand, as drinking progresses, reward networks in the brain seem to become rapidly altered. For example, alcohol-related reward is consistently associated with over-activation of mesolimbic systems relating to reward and motivation (recently reviewed elsewhere [96, 97]), and even teenagers with relatively short histories of AUD have robust reward network response to alcohol images compared with nondrinkers [98]. Taken together, this suggests that difficulty with impulsive responding is an inconsistent risk factor for AUDs, but is more consistently observed as a consequence of both immediate intoxication and heavy drinking histories and/or AUDs. On the other hand, difficulty with impulsive choice has been consistently observed to be both a preexisting risk factor for AUD and a consequence of drinking history, but not a direct consequence of intoxication.

Alcohol-association studies examine how task performance is associated with risk of alcohol use, either by connecting performance to self-report of use, or by attempting to differentiate those at low and high risk. The studies summarized in Table 2 show that, in general, impulsive choice tasks are more consistently associated with risk of alcohol use and problems (although patterns of BART findings are more mixed [99, 100]), whereas impulsive response tasks are less consistently associated (except perhaps among those with more severe disorders). By use of diffusion tensor imaging (DTI), Herting et al. showed that poor white matter integrity through the frontal, temporal, and subcortical pathways mediated the effects of familial alcoholism on slower behavioral responses on a delay discounting task [101]. This could indicate that pre-existing differences in myelination may be the cause of difficulty making decisions among FHP youths. In another DTI study, poorer baseline fronto-limbic white matter integrity was prospectively associated with subsequent risk taking and substance use by adolescents, suggesting that poorer subcortical structural connectivity may be a mechanism underlying the propensity toward risky behavior and involvement with substance use [102].

Although performance on impulsive response tasks usually has little predictive ability, imaging work may suggest otherwise. Specifically, multiple fMRI studies of adolescents with a family history of alcoholism [103] or those who later report heavy drinking [104] have suggested reduced recruitment of regions of the PFC (for example the middle frontal gyrus, the dorsolateral prefrontal, inferior frontal, and ACC) that have been associated with inhibition during go/nogo tasks, even while performance remains equal among groups. Moreover, there is some evidence that neural signatures can predict the development of early impaired control over drinking. Adolescents who later became heavy drinkers or reported blackouts from drinking had a greater frontal PFC response during a no go tasks at baseline assessment before the onset of any drinking, compared with adolescents who did not later report heavy drinking or blackouts [104, 105]. This may reflect over-recruitment of task-relevant regions to compensate for deficient inhibition abilities, and suggests that this neural response may be a marker of risk of later impaired control over drinking.

Finally, behavioral measures also have limitations. They are time-consuming, difficult, and sometimes expensive to administer. Furthermore, their psychometric properties are more difficult to establish by use of conventional methods, because they suffer from the "task impurity problem" (i.e. scores on a task cannot be disaggregated from task-specific demands [106]). Because of fatigue effects, researchers rarely (i.e. only 11 studies reported) include more than one or two measurements of behavior in the same study, making it difficult to achieve convergent or divergent validity or to use latent factor analysis to obtain task-independent estimates of ability [32••]. Many behavioral tasks have only a single summary score, which obviates traditional measures of reliability relying on multiple "items" or scores from a single test (for example split-half reliability or Cronbach's alpha), which, in turn, forces researchers to rely on more costly methods, for example test-retest reliability. Indeed, it is unclear whether behavioral tasks are best categorized as state or trait measures, although this is likely to vary between tasks. Sharma et al. [32••] noted that test-retest reliability of behavioral tasks is 
rare, and often low when tested. However, two recent studies reported moderate to high reliability in several tasks in the very short term (one week to one month), providing some evidence that measures of impulsive choice measure a stable and consistent style (i.e. delay discounting, BART, and probability discounting; one week test-retest $r=0.76-0.89$ ) whereas executive function tasks have a broader range of reliability (i.e. stop-signal, go/no-go, CPT, flanker tasks, antisaccade; one week test-retest $r=0.38-0.92$ ) [107-109]. Finally, a few researchers have begun to use behavioral impulsivity tasks for ecological momentary assessment (EMA) several times per day among smokers and hard drug users. These studies have used variations on the Stroop task $[109,110]$ and the CPT and delay discounting [111] and performance in these studies was affected by cravings and mood, suggesting that performance in these tasks is, at least in part, affected by context. These studies also reported good reliability for these tasks; a cocaine Stroop task, for example, had a reliability of $r=0.68$ [109].

\section{Associations Between Trait and Behavior Measures}

When researchers have attempted to connect trait and behavioral measures of impulsivity they frequently report no, weak, or inconsistent relationships. Several studies have reported no associations [59, 69, 112-114]. One study investigating multiple measures of trait impulsivity using five independent behavioral measures reported only moderate associations with errors in a Stroop task [115], but remained unrelated to all other performance outcomes. A recent meta-analysis of over 100 multi-method studies showed that task measures and selfreport measures, on average, usually had weak correlations (between $r=0.10$ and $r=0.15$ ), although there was variability in the magnitude of the effects [22•]. Another recent metaanalysis reported similar findings for a few task results only [32••]. This could be because of the commonly reported weak association between single instances of behavior and traits, which may improve upon aggregation of multiple measures of behavior [116, 117]. It may also be that conducting behavioral tasks under ideal conditions (for example in a quiet, distraction-free environment), although providing an optimum estimate of ability, may actually not provide an optimum estimate of individual differences in impulsivity, which may, rather, arise only under conditions of challenge or stress. It might only be that individual differences in impulsivity emerge under conditions of stress, fatigue, or negative or positive mood, as they are theorized to do in the real world. Adolescents and young adults with high scores for different aspects of impulsivity do not act impulsively all the time, and understanding the contextual conditions necessary to evoke impulsive behavior may be crucial to observing impulsivity in the laboratory.
Finally, it is possible impulsivity as measured by behavior or trait is simply not the same; this may be an example of the "jingle fallacy" [118]. For example, Sharma et al. [32••] recently reported that impulsivity measured by behavior and trait independently predicts "daily life" outcomes, for example alcohol use and other externalizing behavior. This may not be surprising, given that trait and measures of behavior do not share a common theoretical framework. As can be seen in our review, there is little theoretical overlap between how indicators of behavior and trait measures are organized, and despite its strong theoretical support, few attempts have been made to represent current trait models, for example the UPPS model at the behavioral level. Thus, despite how often authors attempt to use the same or similar labels, it is not clear we can even say that trait and behavior are indicative of the same aspect of impulsivity (see also an excellent meta-analysis by Cyders et al. [119••]). Notable exceptions may include the BART, designed to assess sensitivity to positive reinforcement, and moderately correlated with sensation seeking $(r=0.20-0.35)$ $[65,120,121]$, and the recently developed Maryland resource for the behavioral understanding of reinforcement from negative stimuli (MRBURNS), designed to assess sensitivity to negative reinforcement and correlated with negative urgency $(r=0.26)$ [122]. These somewhat better correlations between trait and behavioral measures suggest that performance on these tasks may better reflect a behavioral manifestation of these impulsive traits, although the trait-like nature of the BART (with test-re-test reliability $>0.75$ ) suggests that it may reflect a separate impulsigenic trait rather than the behavioral manifestation of a trait.

\section{Conclusions}

Most researchers who attempt to characterize the neural basis of impulsivity in alcohol disorders have used behavioral impulsivity tasks to build their phenotype, with some authors going as far as arguing that impulsivity research has moved beyond the use of traits to focus largely on behavioral indicators of impulsivity because traits do not predict behavior [58]. However, it is clear from several reviews that traits, as indicators of impulsivity, are actually, the best predictor of individual differences in alcohol behavior (and, indeed, most behavior) $[28 \bullet, 29 \bullet, 30 \bullet$, although some indicators of behavior seem to have more predictive value [32••]. It is interesting that our neural maps of the risk of alcohol disorders are being built largely without utilizing the single best predictor of alcohol behavior. One promising direction in trait impulsivity research involves the measurement of self-control specific to the drinking context. Impaired control over drinking reflects the inability to stop or limit drinking at will [1] and is regarded as a dysregulated pattern of drinking [7, 8]. This aspect, with 
relationships to enhanced responses to alcohol in the insula, precentral gyrus, and putamen areas of the brain [92•], has recently been identified as understudied $[3 \bullet \bullet$. Thus, understanding the neural basis of individual differences in impulsivity and impulsivity specifically directed toward drinking (on the basis of the three factor framework) at the trait level is a critical topic for future research.

The importance of a coherent, valid hypothesis cannot be overstated. As Strauss and Smith [123] noted, a hypothesis may fail because the relationship between, for example, impulsivity and alcohol use during adolescence does not exist, but also because impulsivity was not measured validly, or that impulsivity encompasses more than one concept in a single measurement, which obscures the true relationship between impulsivity and alcohol use. This is true for some positive findings: a positive finding could also result either from a nonrejected hypothesis or from a measure of impulsivity that mixes two concepts in a single indicator, only one of which is associated with alcohol use. If impulsivity is truly multidimensional, as is becoming increasingly recognized [27, $32 \cdot \bullet$, then using uni-dimensional indicators of impulsivity at the trait or behavioral level [119••] becomes important, especially as many reviews have suggested all aspects of impulsivity are associated with alcohol use and problems [28•, 30•].

Moreover, the use of modern psychometrics to investigate these uni-dimensional structures is critical. Although authors frequently describe impulsivity in terms of a latent concept, they often rely on earlier scales (many versions of which are still commonly used) that were developed by such psychometric approaches as principal-components analysis (PCA) or applications of exploratory factor analysis $[124,125]$ that have since been shown to have the potential to produce misleading solutions (for example orthogonal factor rotations or assuming that binary or ordinal response options can be accurately treated as continuous [126, 127]). For example, most studies establishing the validity of the Barratt impulsiveness scale $[25,128]$ utilized PCA, an approach which has been criticized because it assumes items are measured without error and does not explicitly model a latent concept believed to underlie item responses [124]. On the other hand, more recently developed scales (for example the UPPS, $[26,27]$ ) tend to utilize combinations of exploratory and confirmatory factor analysis. These differences in how trait scales were first developed makes the task of comparing across scales more challenging, because it may be that scales differ in the extent to which they are unitary or multi-dimensional, or what range of severity they cover, or how much information they provide. Although psychometricians recommend testing and reporting scale information beyond simple latent factors, for example tests of invariance across groups (or age), or test information, item difficulty, and discrimination [129, 130], many well established scales lack this information. Without basic psychometrics for common impulsivity scales, it will remain impossible to determine whether different findings from studies arise because they measure different things or because they measure the same thing but do so differently. There are several recent examples, however, for example computer-adaptive administration (CAT, a method which utilizes item-response theory) of the schedule for non-adaptive and adaptive personality (SNAP) [131], or the development of the BIS-brief within an item response theory (IRT) framework [132], or tests of measurement invariance in the UPPS [133], which are excellent examples of how modern psychometric theory might be utilized to construct better indicators as a means of improving the mismeasurement of impulsivity in the field.

We have also noted throughout this paper that there are concepts similar to trait and behavioral impulsivity in the child and adolescent developmental field. Yet the work of researchers who study the development of temperament [33, $39,134]$ and how it relates to precursors of substance use, for example externalizing behavior [39, 40], and adolescent developmental researchers, cited throughout, who study concepts similar to impulsivity (for example "psychosocial maturity", one facet of which is impulse control) and how they are related to substance use and other risk behavior have been, to date, underemphasized.

Moreover, it is important to understand how regions of the brain that are implicated in both impulsivity and alcohol use and problems are related to behavior during development. First, a critical shortcoming of the "developmental asymmetry" model of adolescence is that no studies have shown that individual differences in brain development are associated with individual differences in measures of the traits of sensation seeking or impulse control. Thus, it is critical to understand how existing models of the trait impulsivity may be mapped on to brain development to bridge the brain-behavior gap. Moreover, there has been little work examining whether brain regions responsible for response inhibition in adulthood serve similar functions in adolescence. In general, fMRI studies of response inhibition suggest that adolescents recruit similar areas as adults and perform equally well, yet adolescents engage the dorsolateral prefrontal cortex (DLPFC) more, and other regions less, than adults (reviewed elsewhere [135]), which suggests that cognitive control is more of an effort for adolescents and relies more on a single region of the brain rather than using other functions that also contribute to response inhibition. Silveri et al. [136] demonstrated that young adolescents (age 12-14) have low ACC GABA levels compared with emerging adults. Finally, during an fMRI Stroop task, response in dorsolateral prefrontal "control" regions was less for adolescents than for adults, and this response was associated with measures of impulse control (Weinberger adjustment inventory) and results from the future orientation questionnaire [137]. 
Given that alcohol use and related disorders begin during adolescence and emerging adulthood, and that impulsivity can be observed during childhood and adolescence before the onset of drinking, it is important to think about impulsivity in the context of development. Critical questions include: how does impulsivity, measured as a trait and as a behavior, develop from childhood into adolescence and into adulthood? To what extent is the factor structure of impulsivity consistent during development? And, taken together, how does the development of impulsivity during childhood, adolescence, and young adulthood affect the development of alcohol use and disorders during those same developmental periods?

Development of measures of the state of impulsivity that are clearly connected to the trait, behavioral, and neural models of impulsivity described above is a critical area of future research. This may be achieved by adaptation or alternative use of current measures of behavioral tasks, novel developments in behavioral measurement, or adaptations of measures of traits to the state level. For example, EMA studies utilizing measures of behavioral tasks [109, 110] can provide insight into whether contextual changes affect risk behavior by producing momentary increases in impulsivity. More recently, researchers have attempted to ask participants to self-report their state impulsivity attempts ("I needed to control or fix my mood or thoughts"; Muraven et al. (Muraven, Collins, Shiffman \& Paty, 2005) and another group have developed a brief self-report impulsivity scale designed to be given in an EMA context that is moderately correlated $(r>0.30-42)$ with specific dimensions of both the UPPS and the BIS [138].

An impressive number of treatments have been developed with the specific purpose of improving some aspect of behavioral or cognitive impulsivity as a means of preventing or treating substance use disorders. Recent reviews acknowledge promising evidence of improved executive function from training programs using several methods, including computerized training, meditation, neurofeedback, and physical exercise [139•]; similar work has been conducted among children [140]. Specific to alcohol, working memory training was also associated with greater decreases in alcohol use by heavy drinkers with strong automatic preferences for alcohol [141]. Training in mindfulness results in improvements in impulsivity, including response inhibition, cognitive flexibility, and attention control both for adolescents and adults [142-146], and mindfulness-based intervention has been shown to be effective in the treatment of substance use disorders [147, 148]. However, as one reads this list alone, it is notable how often the "impulsivity" target changes during intervention. Having consistent, clearly, and theoretically derived models of impulsivity as a trait, behavior, and, increasingly, as a state are not only are of benefit to theory but also provide clear and specific behavioral objectives for intervention that spur the development of treatment.

\section{Compliance with Ethics Guidelines}

Conflict of Interest Kevin M. King, Julie A. Patock-Peckham, Alecia D. Dager, Kristine Thimm, and Jonathan R. Gates declare that they have no conflict of interest.

Human and Animal Rights and Informed Consent This article does not contain any studies with human or animal subjects performed by any of the authors.

\section{References}

Papers of particular interest, published recently, have been highlighted as:

- Of importance

•. Of major importance

1. Heather N, Tebbutt JS, Mattick R, Zamir R. Development of a scale for measuring impaired control over alcohol consumption: a preliminary report. J Stud Alcohol. 1993;54:700-9.

2. Association, A.P., Diagnostic and statistical manual of mental disorders 5th ed2013, Arlington, VA: American Psychiatric Publishing.

3.• Leeman RF, Patock-Peckham JA, Potenza MN. Impaired control over alcohol use: an under-addressed risk factor for problem drinking in young adults? Exp Clin Psychopharm. 2012;20:92106. A major review of impaired control over drinking.

4. Koob GF. Neurobiology of addiction. Focus. 2011;9:55-65.

5. Hawkins JD, Catalano RF, Miller JY. Risk and protective factors for alcohol and other drug problems in adolescence and early adulthood: implications for substance abuse prevention. Psychol Bull. 1992;112:64-105.

6. Sher KJ, Trull TJ. Personality and disinhibitory psychopathology: alcoholism and antisocial personality disorder. J Abnorm Psychol. 1994;103:92-102.

7. Patock-Peckham JA, Morgan-Lopez AA. College drinking behaviors: mediational links between parenting styles, impulse control, and alcohol-related outcomes. Psychol Addict Behav. 2006;20:117-25.

8. Patock-Peckham JA, Cheong J, Balhorn ME, Nagoshi CT. A social learning perspective: a model of parenting styles, selfregulation, perceived drinking control, and alcohol Use and problems. Alcohol Clin Exp Res. 2001;25:1284-92.

9. Koob GF, Volkow ND. Neurocircuitry of addiction. Neuropsychopharmacol. 2009;35:217-38.

10. Johnston LD, O'Malley PM, Bachman JG, Schulenberg JE. Monitoring the future national survey results on drug use, 19752012. Volume I: secondary school students. Ann Arbor: Institute for Social Research, The University of Michigan; 2013.

11. United States Department of, H., Human Services. Substance, A., Mental Health Services Administration. Center for Behavioral Health, S., and Quality, National Survey on Drug Use and Health, 2011, 2013, Inter-university Consortium for Political and Social Research (ICPSR) [distributor].

12. Giedd JN, Blumenthal J, Jeffries NO, et al. Brain development during childhood and adolescence: a longitudinal MRI study. Nat Neurosci. 1999;2:861-3.

13. Gogtay N, Giedd JN, Lusk L, et al. Dynamic mapping of human cortical development during childhood through early adulthood. Proc Natl Acad Sci U S A. 2004;101:8174-9.

14. Peters BD, Szeszko PR, Radua J, et al. White matter development in adolescence: diffusion tensor imaging and meta-analytic results. Schizophrenia Bull. 2012;38:1308-17. 
15. Doremus-Fitzwater TL, Varlinskaya EI, Spear LP. Motivational systems in adolescence: possible implications for age differences in substance abuse and other risk-taking behaviors. Brain and cognition. 2010;72:114-23.

16. Lenroot RK, Giedd JN. Brain development in children and adolescents: insights from anatomical magnetic resonance imaging. Neurosci Biobehav Rev. 2006;30:718-29.

17. Steinberg L. A social neuroscience perspective on adolescent risktaking. Dev Rev. 2008;28:78-106

18. Casey B, Jones RM. Neurobiology of the adolescent brain and behavior: implications for substance use disorders. J Am Acad Child Psy. 2010;49:1189-201.

19. Harden KP, Tucker-Drob EM. Individual differences in the development of sensation seeking and impulsivity during adolescence: further evidence for a dual systems model. Dev Psychol. 2011;47: 739-46. One of the first studies to showed that trait measures impulse control and sensation seeking follow predicted trajectories across adolescence and that there are individual differences in typical development.

20. Steinberg L, Graham S, O'Brien L, et al. Age differences in future orientation and delay discounting. Child Dev. 2009;80:28-44.

21. Carver CS. Impulse and constraint: perspectives from personality psychology, convergence with theory in other areas, and potential for integration. Pers Soc Psychol Rev. 2005;9:312-33.

22. Duckworth AL, Kern ML. A meta-analysis of the convergent validity of self-control measures. J Res Pers. 2011;45:259-68. Important cross-disciplinary meta-analysis of multiple trait and behavioral measures of impulsivity.

23. Eysenck SB, Eysenck HJ. Impulsiveness and venturesomeness: their position in a dimensional system of personality description. Psychol Rep. 1978;43:1247-55.

24. Carver CS, Scheier MF. Attention and self-regulation: a controltheory approach to human behavior. New York: Springer; 1981.

25. Patton JH, Stanford MS. Factor structure of the Barratt impulsiveness scale. J Clin Psychol. 1995;51:768-74.

26. Smith GT, Fischer S, Cyders MA, et al. On the validity and utility of discriminating among impulsivity-like traits. Assessment. 2007;14:155-70.

27. Whiteside SP, Lynam DR. The five factor model and impulsivity: using a structural model of personality to understand impulsivity. Pers Indiv Differ. 2001;30:669-89.

28. Coskunpinar A, Dir AL, Cyders MA. Multidimensionality in impulsivity and alcohol Use: a meta-analysis using the UPPS model of impulsivity. Alcohol Clin Exp Res. 2013;37:1441-50. Major meta analysis of the relation between facets of impulsivity and alcohol use.

29. de Ridder DT, Lensvelt-Mulders G, Finkenauer C, et al. Taking stock of self-control a meta-analysis of How trait self-control relates to a wide range of behaviors. Pers Soc Psychol Rev. 2012;16:76-99. Large meta-analysis of multiple measures of trait impulsivity and multiple problem outcomes.

30. Stautz K, Cooper A. Impulsivity-related personality traits and adolescent alcohol use: a meta-analytic review. Clin Psychol Rev. 2013;33:574-92. Large meta analysis of the association between impulsive traits and alcohol use in adolescence.

31.• Cross CP, Copping LT, Campbell A. Sex differences in impulsivity: a meta-analysis. Psychol Bull. 2011;137:97-130. Meta analysis of sex differences in impulsivity, with particularly excellent theoretical coverage of different approaches to measuring and conceptualizing impulsivity.

32.• Sharma, L.M., K.E. \& Clark, L.A.: Toward a theory of distinct types of "Impulsive" behaviors: A meta-analysis of self-report and behavioral measures. Psychol Bull 2013, In press. Meta-analysis of trait and behavioral measures of impulsivity, suggesting convergence across measures into a 3 factor trait structure. Factor analyzes beahvioral impulsivity measures and suggests a 3 factor structure that is different than what is typically proposed in the theoretical literature.

33. Rothbart MK, Ahadi SA, Evans DE. Temperament and personality: origins and outcomes. J Pers Soc Psychol. 2000;78:122-35.

34. Gullo MJ, Dawe S. Impulsivity and adolescent substance use: rashly dismissed as "all-bad"? Neurosci Biobehav R. 2008;32: 1507-18.

35. Gray JA, McNaughton N. The neuropsychology of anxiety: an enquiry into the function of the septo-hippocampal system. 2nd ed. New York: Oxford Science Publications; 2000.

36. Gjedde A, Kumakura Y, Cumming P, et al. Inverted-U-shaped correlation between dopamine receptor availability in striatum and sensation seeking. Proc Natl Acad Sci U S A. 2010;107:3870-5.

37. Zald DH, Cowan RL, Riccardi P, et al. Midbrain dopamine receptor availability is inversely associated with novelty-seeking traits in humans. J Neurosci. 2008;28:14372-8.

38. DeYoung CG, Hirsh JB, Shane MS, et al. Testing predictions from personality neuroscience brain structure and the big five. Psychol Sci. 2010;21:820-8. Reports connections between the volume of specific brain structures and individual differences in trait personality, including Extraversion and Neuroticism.

39. Eisenberg N, Spinrad TL, Fabes RA, et al. The relations of effortful control and impulsivity to children's resiliency and adjustment. Child Dev. 2004;75:25-46.

40. Lengua LJ, Bush NR, Long AC, et al. Effortful control as a moderator of the relation between contextual risk factors and growth in adjustment problems. Dev Psychopathol. 2008;20: 509-28.

41. Wills TA, Sandy JM, Yaeger AM. Time perspective and earlyonset substance use: a model based on stress-coping theory. Psychol Addict Behav. 2001;15:118-25.

42. Wills TA, Stoolmiller M. The role of self-control in early escalation of substance use: a time-varying analysis. J Consult Clin Psych. 2002;70:986-97.

43. Zimbardo PG, Boyd JN. Putting time in perspective: a valid, reliable individual-differences metric. J Pers Soc Psychol. 1999;77:1271-88.

44. Weinberger, D.: Social-emotional adjustment in older children and adults: I. Psychometric properties of the Weinberger Adjustment Inventory. Unpublished manuscript 1991.

45. Boes AD, Bechara A, Tranel D, et al. Right ventromedial prefrontal cortex: a neuroanatomical correlate of impulse control in boys. Soc Cogn Affect Neurosci. 2009;4:1-9.

46. Wills TA, Dishion TJ. Temperament and adolescent substance use: a transactional analysis of emerging self-control. J Clin Child Adolesc. 2004;33:69-81.

47. Smillie LD, Jackson CJ, Dalgleish LI, Conceptual distinctions among Carver and White's. BAS scales: a reward-reactivity versus trait impulsivity perspective. Pers Indiv Differ. 1994;2006(40): 1039-50.

48. Boy F, Evans CJ, Edden RA, et al. Dorsolateral prefrontal $\gamma$ aminobutyric acid in men predicts individual differences in rash impulsivity. Biol Psychiat. 2011;70:866-72.

49. Bjørnebekk A, Fjell AM, Walhovd KB, et al. Neuronal correlates of the five factor model (FFM) of human personality: multimodal imaging in a large healthy sample. Neuroimage. 2013;65:194-208.

50. Bechara A. Decision making, impulse control and loss of willpower to resist drugs: a neurocognitive perspective. Nat Neurosci. 2005;8:1458-63.

51. Casey B, Jones RM, Hare TA. The adolescent brain. Ann NY Acad Sci. 2008;1124:111-26. Describes neurobiological models of adolescent development and how it may influence risk for substance use disorders.

52. Monahan KC, Steinberg L, Cauffman E, Mulvey EP. Trajectories of antisocial behavior and psychosocial maturity from adolescence to young adulthood. Dev Psychol. 2009;45:1654-68. 
53. Sher KJ, Grekin ER, Williams NA. The development of alcohol use disorders. Ann Rev Clin Psychol. 2005;1:493-523.

54.• Winstanley CA, Olausson P, Taylor JR, Jentsch JD. Insight into the relationship between impulsivity and substance abuse from studies using animal models. Alcohol Clin Exp Res. 2010;34: 1306-18. Excellent review describing converging evidence across animal and human models for how behavioral models can be both predictive of and influenced by substance use across the cycle of the development of addiction.

55. Dougherty DM, Mathias CW, Marsh-Richard DM, et al. Distinctions in behavioral impulsivity: implications for substance abuse research. Addict Disord Their Treat. 2009;8:61-73.

56. MacLeod CM. Half a century of research on the stroop effect: an integrative review. Psychol Bull. 1991;109:163-203.

57. Munoz DP, Everling S. Look away: the anti-saccade task and the voluntary control of eye movement. Nat Rev Neuro. 2004;5: 218-28.

58. Caswell AJ, Morgan MJ, Duka T. Inhibitory control contributes to "motor"-but not "cognitive"-impulsivity. Exp Psychol. 2013;60: 324-34.

59. Reynolds B, Ortengren A, Richards JB, de Wit H. Dimensions of impulsive behavior: Personality and behavioral measures. Pers Indiv Differ. 2006;40:305-15.

60. Potenza MN, De Wit H. Control yourself: alcohol and impulsivity. Alcohol Clin Exp Res. 2010;34:1303-5.

61. Giancola PR, Tarter RE. Executive cognitive functioning and risk for substance abuse. Psychol Sci. 1999;10:203-5.

62. Green L, Fry AF, Myerson J. Discounting of delayed rewards: a life-span comparison. Psychol Sci. 1994;5:33-6.

63. Buelow MT, Suhr JA. Construct validity of the Iowa gambling task. Neuropsychol Rev. 2009;19:102-14.

64. Figner B, Mackinlay RJ, Wilkening F, Weber EU. Affective and deliberative processes in risky choice: age differences in risk taking in the Columbia card task. J Exp Psychol Learn. 2009;35: 709-30.

65. Lejuez C, Read JP, Kahler CW, et al. Evaluation of a behavioral measure of risk taking: the balloon analogue risk task (BART). J Exp Psychol-Appl. 2002;8:75.

66. Verdejo-García A, Lawrence AJ, Clark L. Impulsivity as a vulnerability marker for substance-use disorders: review of findings from high-risk research, problem gamblers and genetic association studies. Neurosci Biobehav R. 2008;32:777-810.

67. Bechara A, Van Der Linden M. Decision-making and impulse control after frontal lobe injuries. Curr Opin Neurol. 2005;18:734-9.

68. Dick DM, Smith G, Olausson P, et al. Review: understanding the construct of impulsivity and its relationship to alcohol use disorders. Addict Biol. 2010;15:217-26. Trait, behavioral and neurobiologial review of the relation between impulsivity and alcohol use disorders.

69. Lane SD, Cherek DR, Rhoades HM, et al. Relationships among laboratory and psychometric measures of impulsivity: implications in substance abuse and dependence. Addict Disord Their Treat. 2003;2:33-40.

70. Reynolds B, Penfold RB, Patak M. Dimensions of impulsive behavior in adolescents: laboratory behavioral assessments. Exp Clin Psychopharm. 2008;16:124.

71. Acheson A, Richard DM, Mathias CW, Dougherty DM. Adults with a family history of alcohol related problems are more impulsive on measures of response initiation and response inhibition. Drug Alcohol Depen. 2011;117:198-203.

72. Mathias CW, Marsh-Richard DM, Dougherty DM. Behavioral measures of impulsivity and the Law. Behav Sci Law. 2008;26: 691-707.

73. Reynolds B, Richards JB, de Wit H. Acute-alcohol effects on the experiential discounting task (EDT) and a question-based measure of delay discounting. Pharmacol Biochem Be. 2006;83:194-202.
74. Dougherty DM, Bjork JM, Andrew Harper R, et al. Behavioral impulsivity paradigms: a comparison in hospitalized adolescents with disruptive behavior disorders. J Child Psychol Pyc. 2003;44: $1145-57$.

75. Dougherty DM, Marsh DM, Mathias CW, et al. The effects of alcohol on laboratory-measured impulsivity after L-tryptophan depletion or loading. Psychopharmacology. 2007;193:137-50.

76. Dougherty DM, Moeller FG, Steinberg JL, et al. Alcohol increases commission error rates for a continuous performance test. Alcohol Clin Exp Res. 1999;23:1342-51.

77. Zhou Q, Chen SH, Main A. Commonalities and differences in the research on Children's effortful control and executive function: a call for an integrated model of self-regulation. Child Dev Perspect. 2012;6:112-21.

78. Fan J, Flombaum JI, McCandliss BD, et al. Cognitive and brain consequences of conflict. Neuroimage. 2003;18:42-57.

79. Dalley JW, Everitt BJ, Robbins TW. Impulsivity, compulsivity, and top-down cognitive control. Neuron. 2011;69:680-94.

80. Aron AR. From reactive to proactive and selective control: developing a richer model for stopping inappropriate responses. Biol Psychiat. 2011;69:e55-68.

81. Casey B, Epstein J, Buhle J, et al. Frontostriatal connectivity and its role in cognitive control in parent-child dyads with ADHD. Am J Psychiat. 2007;164:1729-36.

82. Chatham CH, Claus ED, Kim A, et al. Cognitive control reflects context monitoring, not motoric stopping, in response inhibition. PLOS One. 2012; 7:e31546.

83.• Miyake A, Friedman NP. The nature and organization of individual differences in executive functions four general conclusions. Curr Dir Psychol Sci. 2012;21:8-14. Review of executive function research, concludes that inhibition fits best not as a unique factor but rather as a consequence of broad ECF.

84. Munakata Y, Herd SA, Chatham CH, et al. A unified framework for inhibitory control. Trends Cogn Sci. 2011;15:453-9. Describes an alternative theory for inhibition as one of competition between alterative goals rather than global stopping.

85. Friedman NP, Miyake A, Young SE, et al. Individual differences in executive functions are almost entirely genetic in origin. J Exp Psychol Gen. 2008;137:201-25.

86. Bridgett DJ, Oddi KB, Laake LM, et al. Integrating and differentiating aspects of self-regulation: effortful control, executive functioning, and links to negative affectivity. Emotion. 2013;13:47.

87. Bechara A, Damasio H, Damasio AR. Emotion, decision making and the orbitofrontal cortex. Cerebral cortex. 2000;10:295-307.

88. Bjork JM, Momenan R, Hommer DW. Delay discounting correlates with proportional lateral frontal cortex volumes. Biol Psychiat. 2009;65:710-3.

89. Bjork JM, Smith AR, Danube CL, Hommer DW. Developmental differences in posterior mesofrontal cortex recruitment by risky rewards. J Neurosci. 2007;27:4839-49.

90. Crowley TJ, Dalwani MS, Mikulich-Gilbertson SK, et al. Risky decisions and their consequences: neural processing by boys with antisocial substance disorder. PLOS One. 2010;5:e12835.

91. Rao H, Korczykowski M, Pluta J, et al. Neural correlates of voluntary and involuntary risk taking in the human brain: an fMRI Study of the Balloon Analog Risk Task (BART). Neuroimage. 2008;42:902-10.

92. Claus ED, Hutchison KE. Neural mechanisms of risk taking and relationships with hazardous drinking. Alcohol Clin Exp Res. 2012;36:408-16. Shows impaired control over drinking maps onto neural models.

93. Bogg T, Fukunaga R, Finn PR, Brown JW. Cognitive control links alcohol use, trait disinhibition, and reduced cognitive capacity: evidence for medial prefrontal cortex dysregulation during reward-seeking behavior. Drug Alcohol Depend. 2012;122:112-8. 
94. - Wetherill, R.R., Squeglia, L.M., Yang, T.T., and Tapert, S.F.: A longitudinal examination of adolescent response inhibition: neural differences before and after the initiation of heavy drinking. Psychopharmacology 2013. Suggests that response inhibition is both altered prior to the onset of heavy drinking for adolescents, and altered by heavy drinking itself.

95. Ahmadi, A., Pearlson, G.D., Meda, S.A., et al.: Influence of Alcohol Use on Neural Response to Go/No-Go Task in College Drinkers. Neuropsychopharmacol 2013.

96. Heinz A, Beck A, Grüsser SM, et al. Identifying the neural circuitry of alcohol craving and relapse vulnerability. Addict Biol. 2009;14:108-18.

97. Schacht JP, Anton RF, Myrick H. Functional neuroimaging studies of alcohol cue reactivity: a quantitative meta-analysis and systematic review. Addic Biol. 2013;18:121-33.

98. Tapert SF, Cheung EH, Brown GG, et al. Neural response to alcohol stimuli in adolescents with alcohol use disorder. Arch Gen Psychiat. 2003;60:727-35.

99. Fernie G, Cole JC, Goudie AJ, Field M. Risk-taking but not response inhibition or delay discounting predict alcohol consumption in social drinkers. Drug Alcohol Depend. 2010;112:54-61.

100. Skeel RL, Pilarski C, Pytlak K, Neudecker J. Personality and performance-based measures in the prediction of alcohol use. Psychol Addict Behav. 2008;22:402-9.

101. Herting MM, Schwartz D, Mitchell SH, Nagel BJ. Delay discounting behavior and white matter microstructure abnormalities in youth with a family history of alcoholism. Alcohol Clin Exp Res. 2010;34:1590-602.

102. Jacobus J, Thayer RE, Trim RS, et al. White matter integrity, substance Use, and risk taking in adolescence. 2012.

103. Schweinsburg AD, Paulus MP, Barlett VC, et al. An fMRI study of response inhibition in youths with a family history of alcoholism. Ann NY Acad Sci. 2004;1021:391-4.

104. Norman AL, Pulido C, Squeglia LM, et al. Neural activation during inhibition predicts initiation of substance use in adolescence. Drug Alcohol Depend. 2011;119:216-23.

105. Wetherill RR, Castro N, Squeglia LM, Tapert SF. Atypical neural activity during inhibitory processing in substance-naive youth who later experience alcohol-induced blackouts. Drug Alcohol Depen. 2013;128:243-9.

106. Friedman NP, Miyake A, Robinson JL, Hewitt JK. Developmental trajectories in toddlers' self-restraint predict individual differences in executive functions 14 years later: a behavioral genetic analysis. Dev Psychol. 2011;47:1410-30.

107. Weafer, J., Baggott, M.J., and de Wit, H.: Test-Retest Reliability of Behavioral Measures of Impulsive Choice, Impulsive Action, and Inattention. Exp Clin Psychopharm 2013.

108. Wöstmann NM, Aichert DS, Costa A, et al. Reliability and plasticity of response inhibition and interference control. Brain and cognition. 2013;81:82-94.

109. Waters AJ, Marhe R, Franken IH. Attentional bias to drug cues is elevated before and during temptations to use heroin and cocaine. Psychopharmacology. 2012;219:909-21.

110. Waters AJ, Li Y. Evaluating the utility of administering a reaction time task in an ecological momentary assessment study. Psychopharmacology. 2008;197:25-35.

111. McCarthy, D.E., EMA of Treatment Mediators and Proximal Predictors of Smoking During a Quit Attempt, 2012, SNRT.

112. Bachorowski J-A, Newman JP. Impulsivity in adults: Motor inhibition and time-interval estimation. Pers Indiv Differ. 1985;6:133-6.

113. Franken IH, Muris P. Individual differences in decision-making. Pers Indiv Differ. 2005;39:991-8.

114. Wingrove J, Bond AJ. Impulsivity: A state as well as trait variable. Does mood awareness explain low correlations between trait and behavioural measures of impulsivity? Pers Indiv Differ. 1997;22: 333-9.
115. Enticott PG, Ogloff JR, Bradshaw JL. Associations between laboratory measures of executive inhibitory control and self-reported impulsivity. Pers Indiv Differ. 2006;41:285-94.

116. Kenrick DT, Funder DC. Profiting from controversy: Lessons from the person-situation debate. Am Psychol. 1988;43:23-34.

117. Rushton JP, Brainerd CJ, Pressley M. Behavioral development and construct validity: The principle of aggregation. Psychol Bull. 1983;94:18-38.

118. Kelley TL. Interpretation of educational measurements. 1927.

119.• Cyders MA, Coskunpinar A. Measurement of constructs using self-report and behavioral lab tasks: Is there overlap in nomothetic span and construct representation for impulsivity? Clin Psychol Rev. 2011;31:965-82. An outstanding review and meta-anlaysis of trait and behavioral measurement of impulsivity, concluding that we often do not observe concurrence across trait and behavioral measures of impulsivity because they were not (despite similar construct labels) designed to assess parallel constructs.

120. Lejuez C, Aklin WM, Zvolensky MJ, Pedulla CM. Evaluation of the balloon analogue risk task (BART) as a predictor of adolescent real-world risk-taking behaviours. J Adolescence. 2003;26:475-9.

121. Lejuez C, Aklin W, Daughters S, et al. Reliability and validity of the youth version of the balloon analogue risk task (BART-Y) in the assessment of risk-taking behavior among inner-city adolescents. J Clin Child Adolesc. 2007;36:106-11.

122. MacPherson L, Calvin NT, Richards JM, et al. Development and preliminary validation of a behavioral task of negative reinforcement underlying risk-taking and its relation to problem alcohol Use in college freshmen. Alcohol Clin Exp Res. 2012;36:426-33.

123. Strauss ME, Smith GT. Construct validity: advances in theory and methodology. Ann Rev Clin Psychol. 2009;5:1-25.

124. Fabrigar LR, Wegener DT, MacCallum RC, Strahan EJ. Evaluating the use of exploratory factor analysis in psychological research. Psychol Methods. 1999;4:272-99.

125. Ford JK, MacCallum RC, Tait M. The application of exploratory factor analysis in applied psychology: a critical review and analysis. Pers Psychol. 1986;39:291-314.

126. Flora DB, Curran PJ. An empirical evaluation of alternative methods of estimation for confirmatory factor analysis with ordinal data. Psychol Methods. 2004;9:466-91.

127. Waller, N.G.: Searching for structure in the MMPI. In The new rules of measurement: What every psychologist and educator should know, Edited by, Mahwah, NJ: Erlbaum. 1999: 185-217.

128. Barratt ES, Stanford MS, Dowdy L, et al. Impulsive and premeditated aggression: a factor analysis of self-reported acts. Psychiat Res. 1999;86:163-73.

129. Borsboom D. The attack of the psychometricians. Psychometrika. 2006;71:425-40.

130. Millsap RE. Invariance in measurement and prediction: their relationship in the single-factor case. Psychol Methods. 1997;2: 248-60.

131. Simms LJ, Clark LA. Validation of a computerized adaptive version of the schedule for nonadaptive and adaptive personality (SNAP). Psychol Assess. 2005;17:28-43.

132. Steinberg L, Sharp C, Stanford MS, Tharp AT. New tricks for an old measure: the development of the Barratt impulsiveness scalebrief (BIS-brief). Psychol Assess. 2013;25:216-26.

133. Cyders MA. Impulsivity and the sexes measurement and structural invariance of the UPPS-P impulsive behavior scale. Assessment. 2013;20:86-97.

134. Rueda, M.R., Posner, M.I., and Rothbart, M.K.: Attentional control and self-regulation. In Handbook of self-regulation: Research, theory, and applications, 2004: 284-299.

135. Luna B, Padmanabhan A, O'Hearn K. What has fMRI told us about the development of cognitive control through adolescence? Brain and cognition. 2010;72:101-13. 
136. Silveri, M.M., Sneider, J.T., Crowley, D.J., et al.: Frontal lobe $\gamma$ aminobutyric acid levels during adolescence: associations with impulsivity and response inhibition. Biol Psychiat 2013.

137. Andrews-Hanna JR, Seghete KLM, Claus ED, et al. Cognitive control in adolescence: neural underpinnings and relation to selfreport behaviors. PLOS One. 2011;6:e21598.

138. Tomko, R., Solhan, M., Carpenter, R., et al.: Measuring Impulsivity in Daily Life: The Momentary Impulsivity Scale. Psychol Assess 2013.

139. Rabipour S, Raz A. Training the brain: fact and fad in cognitive and behavioral remediation. Brain and cognition. 2012;79:15979. Major review of cognitive training paradigms.

140. Diamond A, Lee K. Interventions shown to aid executive function development in children 4 to 12 years old. Science. 2011;333: 959-64.

141. Houben K, Wiers RW, Jansen A. Getting a grip on drinking behavior training working memory to reduce alcohol abuse. Psychol Sci. 2011;22:968-75.

142. Chambers R, Lo BCY, Allen NB. The impact of intensive mindfulness training on attentional control, cognitive style, and affect. Cognitive Ther Res. 2008;32:303-22.

143. Oberle E, Schonert-Reichi KA, Lawlor MS, Thomson KC. Mindfulness and inhibitory control in early adolescence. J Early Adolescence. 2012;32:565-88.

144. Sahdra BK, MacLean KA, Ferrer E, et al. Enhanced response inhibition during intensive meditation training predicts improvements in self-reported adaptive socioemotional functioning. Emotion. 2011;11:299-312.

145. Tang YY, Ma Y, Wang J, et al. Short-term meditation training improves attention and self-regulation. Proc Natl Acad Sci U S A. 2007;104:17152-6.

146. Zylowska L, Ackerman DL, Yang MH, et al. Mindfulness meditation training in adults and adolescents with ADHD: a feasibility study. J Atten Disord. 2008;11:737-46.

147. Bowen S, Chawla N, Marlatt GA. Mindfulness-based relapse prevention for addictive behaviors: a clinician's guide. New York: The Guilford Press; 2011.

148. Witkiewitz K, Marlatt GA, Walker DD. Mindfulness-based relapse prevention for alcohol and substance use disorders. J Cognit Psychother. 2005;19:211-28.

149. Carver CS, White TL. Behavioral inhibition, behavioral activation, and affective responses to impending reward and punishment: The BIS/BAS Scales. J Pers Soc Psychol. 1994;67:319-33.

150. Patrick CJ, Curtin JJ, Tellegen A. Development and validation of a brief form of the multidimensional personality questionnaire. Psychol Assess. 2002;14:150-63.

151. Eysenck SB, Eysenck HJ, Barrett P. A revised version of the psychoticism scale. Pers Indiv Differ. 1985;6:21-9.

152. Dickman SJ. Functional and dysfunctional impulsivity: personality and cognitive correlates. J Pers Soc Psychol. 1990;58:95-102.

153. Buss AH, Plomin R. Temperament: early developing personality traits. Hillsdale: Erlbaum; 1984.
154. McCrae RR, Costa PT. Validation of the five-factor model of personality across instruments and observers. J Pers Soc Psychol. 1987;52:81-90.

155. Dougherty DM, Marsh-Richard DM, Hatzis ES, et al. A test of alcohol dose effects on multiple behavioral measures of impulsivity. Drug Alcohol Depend. 2008;96:111-20.

156. Corbin WR, Cronce JM. Alcohol effects on behavioral control: the impact of likelihood and magnitude of negative consequences. Alcohol Clin Exp Res. 2007;31:955-64.

157. Curtin JJ, Fairchild BA. Alcohol and cognitive control: implications for regulation of behavior during response conflict. J Abnorm Psychol. 2003;112:424-36.

158. David IA, Volchan E, Alfradique I, et al. Dynamics of a stroop matching task: effect of alcohol and reversal with training. Psychol Neurosci. 2011;4:279-83.

159. Fillmore MT, Vogel-Sprott M. An alcohol model of impaired inhibitory control and its treatment in humans. Exp Clin Psychopharm. 1999;7:49-55.

160. Courtney KE, Arellano R, Barkley-Levenson E, et al. The relationship between measures of impulsivity and alcohol misuse: an integrative structural equation modeling approach. Alcohol Clin Exp Res. 2012;36:923-31.

161. Goudriaan AE, Grekin ER, Sher KJ. Decision making and binge drinking: a longitudinal study. Alcohol Clin Exp Res. 2007;31: 928-38.

162. Salgado JV, Malloy-Diniz LF, Campos VR, et al. Neuropsychological assessment of impulsive behavior in abstinent alcohol-dependent subjects. Rev Bras Psiquiatr. 2009;31:4-9.

163. Peacock A, Bruno R, Martin FH, Carr A. The impact of alcohol and energy drink consumption on intoxication and risk-taking behavior. Alcohol Clin Exp Res. 2013;37:1234-42.

164. Bidwell L, MacKillop J, Murphy JG, et al. Biphasic effects of alcohol on delay and probability discounting. Exp Clin Psychopharm. 2013;21:214-21.

165. McCarthy DM, Niculete ME, Treloar HR, et al. Acute alcohol effects on impulsivity: associations with drinking and driving behavior. Addiction. 2012;107:2109-14.

166. Euser AS, Van Meel CS, Snelleman M, Franken IH. Acute effects of alcohol on feedback processing and outcome evaluation during risky decision-making: an ERP study. Psychopharmacology. 2011;217:111-25.

167. Field M, Christiansen P, Cole J, Goudie A. Delay discounting and the alcohol stroop in heavy drinking adolescents. Addiction. 2007;102:579-86.

168. Petry NM. Delay discounting of money and alcohol in actively using alcoholics, currently abstinent alcoholics, and controls. Psychopharmacology. 2001;154:243-50.

169. Barry D, Petry NM. Predictors of decision-making on the Iowa gambling task: independent effects of lifetime history of substance use disorders and performance on the trail making test. Brain Cognition. 2008;66:243-52. 